\title{
Dynamics of DFB dye lasing by polarization modulation: simulations and experiment
}

\author{
Denis V Novitsky, Vasili M Katarkevich and Terlan Sh \\ Efendiev \\ B. I. Stepanov Institute of Physics, National Academy of Sciences of Belarus, \\ Nezavisimosti Avenue 68, BY-220072 Minsk, Belarus \\ E-mail: dvnovitsky@gmail.com
}

\begin{abstract}
Distributed feedback (DFB) dye lasing by polarization modulation is studied theoretically on the basis of modified rate equations. Numerical solution of these equations allows to obtain the generated power dynamics and the dependence of laser energy on pump energy. The results of calculations are in good qualitative agreement with the experimental data.

PACS numbers: 42.55.Mv, 42.65.Re
\end{abstract}

Keywords: DFB dye laser, polarization grating, rate equations

Submitted to: Laser Phys. Lett. 


\section{Introduction}

Distributed feedback (DFB) lasers are compact and easy-to-use sources of the narrowband coherent radiation. In the DFB lasers, the feedback is provided via backward Bragg light scattering from the spatially periodic structure formed inside the active medium itself and, hence, is distributed throughout its length. The idea of such lasers has been put forward and implemented by the authors of Refs. [1, 2]. As applied to dye solutions as active media, the spatially periodic structures can be either of stationary [1] or dynamic (rapidly reversible) 2] character. It should be noted that the dynamic DFB dye lasers exhibit the highest performance characteristics. A number of optical schemes of such lasers were developed. Their advantages had been demonstrated, such as a small spectral linewidth, wide tuning range, high efficiency, possibility of the high repetition rate operation without dye solution flow as well as simultaneous generation at two or more wavelengths with independent spectral tuning, etc. [3, 4, 5, 6, 7, 8, 9, 10, 11. At the same time, the most interesting and important feature of the dynamic DFB dye lasers is their ability to produce picosecond pulses both at picosecond and nanosecond excitation [12, 13, 14, 15, 16, 17, 18. In the latter case, the use of such lasers represents the simplest way for obtaining single pulses of a few tens of picoseconds duration with possibility of smooth wavelength tuning.

Due to their important merits, DFB dye lasers are still the subject of steady attention and intensive study by scientists from different research centers (see some recent publications [19, 20, 21, 22, 23, 24, 25, 26]).

In the dynamic DFB dye lasers, excitation of the active medium is generally provided by two converging pump beams with vertical orientation of the electric field vector (s-polarization). In this case, spatially periodic modulation of the resultant pump field intensity is provided along the excited dye region. Fundamentally different type of modulation occurs when the pump beams are orthogonally polarized (for example, the first one is s-polarized, while the second one is p-polarized). Under such conditions, the pump field intensity is uniform over the excited zone of the dye, while a periodic change of the resultant excitation field polarization takes place. Due to the anisotropy of light absorption and emission by dye molecules, the above pump field polarization modulation results in the formation of a transient gain dichroism (i.e., polarization) grating inside the dye solution.

Dynamic DFB dye laser action by polarization modulation was experimentally investigated in Refs. [27, 28, 29, 30] where spectral, threshold and polarization characteristics of a DFB laser pumped by the second harmonic from a nanosecond Nd:YAG laser were reported. Unlike the works mentioned above, in our studies we used the second harmonic generation from a subnanosecond diode-pumped solidstate (DPSS) Nd:LSB microlaser to initiate the DFB lasing by polarization modulation in the dye solution [31, 32. Under such condition, the DFB laser exhibited a spectral linewidth of $\Delta \lambda_{0.5}<0.008 \mathrm{~nm}$ (full width at half-maximum, FWHM) and the energy conversion efficiency as high as $\eta_{\max } \sim 48 \%$. It should be noted for comparison that, under identical pumping conditions, the DFB lasing by intensity modulation revealed the similar value of $\Delta \lambda_{0.5}$ and $\eta_{\max } \sim 50 \%$. Thus, in terms of the spectral linewidth and peak lasing efficiency, the DFB laser based on the dynamic polarization grating is comparable with that employing the "traditional" (i.e., intensity modulation) pumping geometry. To the best of our knowledge, no similar results regarding spectral line narrowness and efficiency of the DFB dye lasing by polarization modulation have been reported ever before. At the same time, our results seem to be of an undoubted interest and indicate the potential of these devices. It is obvious that, for its practical realization, a deeper insight into the operation of such DFB dye lasers is required. The latter is hardly possible without appropriate theoretical model capable to adequately predict their performance. Since, as far as we know, all existing studies still lack for any theoretical treatment of the properties of such laser devices, here we attempt to fill this gap.

In this paper, we propose a simple theoretical model of DFB lasing by polarization modulation and report on the numerical simulations of its dynamics for the case of subnanosecond excitation. The results obtained reveal that, at moderate pumping rates, a multi-spike emission is provided by a DFB laser, while single picosecond pulse generation takes place at the pump intensities not far from the threshold. As a proof of consistency and usefulness of the proposed model, the output characteristics of the Rhodamine 6G DFB laser excited by two orthogonally polarized second harmonic beams from a subnanosecond DPSS Nd:LSB microlaser are presented. 


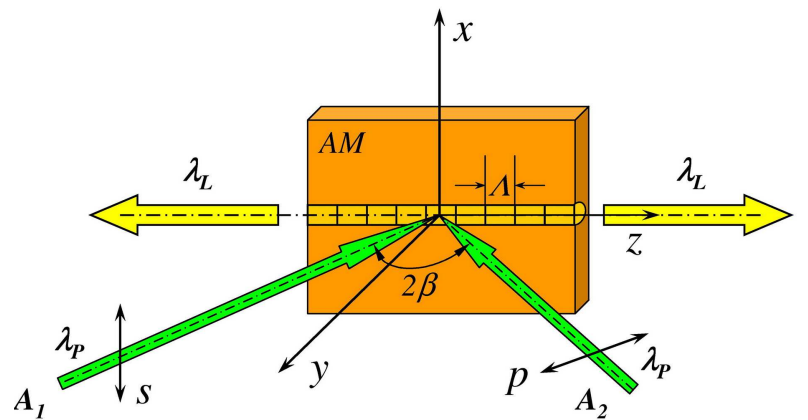

Figure 1. (Colour online) Schematic of the DFB laser excitation by two crossing orthogonally polarized pumping beams. AM, active medium; $\lambda_{P}$, pumping wavelength; $\lambda_{L}$, lasing wavelength; $2 \beta$, intersection angle of the pump beams inside the medium; $\Lambda$, spatial modulation period; $A_{1}$ and $A_{2}$, amplitudes of the pump waves.

\section{Theoretical model}

The geometry of the model is shown in Fig. 1. The field of the pump beam which forms DFB grating is a sum of two plane waves crossing inside the medium at an angle $2 \beta$. We denote $\beta$ the angle between the pump wave vector and the normal to the surface of the sample (plane $x-z$ ). We assume that the first pump wave is polarized along $x$ axis (s-polarization), the second one is polarized in the plane $y-z$ (p-polarization), and the wave generated by the laser propagates along $z$ axis. Then, spatial distribution of the total pump electric field can be written (after time averaging) as follows,

$$
\begin{aligned}
\vec{E} & =\vec{e}_{x} A_{1} \mathrm{e}^{-\mathrm{i} k(y \cos \beta+z \sin \beta)}+ \\
& +\left(\vec{e}_{y} \sin \beta+\vec{e}_{z} \cos \beta\right) A_{2} \mathrm{e}^{-\mathrm{i} k(y \cos \beta-z \sin \beta)},
\end{aligned}
$$

where $A_{1}$ and $A_{2}$ are the amplitudes of the pump waves, $\vec{e}_{x, y, z}$ are the unit vectors along the corresponding axes, $k=2 \pi / \lambda_{P}$ is the wavenumber of the pump waves with the wavelength $\lambda_{P}$. The field (11) gives the periodic change of the polarization state along $z$ axis, while its intensity $|\vec{E}|^{2}$ does not depend on position.

Interaction of a dye molecule with the field is governed by the value of $|\vec{d} \cdot \vec{E}|^{2}$, the direction of the molecular dipole moment vector being defined as

$\vec{d}=|d|\left(\vec{e}_{x} \sin \theta \cos \varphi+\vec{e}_{y} \cos \theta \sin \varphi+\vec{e}_{z} \cos \theta\right)$,

where the angles of the spherical coordinate system $\theta$ (zenith angle) and $\varphi$ (azimuth angle) are counted from $z$ and $x$ axes, correspondingly. Using Eqs. (1) and (2), the excitation efficiency for the molecule with dipole moment oriented along angles $\theta$ and $\varphi$ can be calculated by

$$
\begin{aligned}
f_{\operatorname{exc}}(\theta, \varphi) & =|\vec{d} \cdot \vec{E}|^{2} /|\vec{d}|^{2}|\vec{E}|^{2}= \\
& =A(\theta, \varphi)+B(\theta, \varphi) \cos (2 \pi z / \Lambda) .
\end{aligned}
$$

Here $\Lambda=\pi / k \sin \beta=\lambda_{P} / 2 \sin \beta$ is the grating period, and the auxiliary functions are

$$
\begin{aligned}
A(\theta, \varphi) & =I_{1} \sin ^{2} \theta \cos ^{2} \varphi+I_{2}\left(\sin ^{2} \beta \sin ^{2} \theta \sin ^{2} \varphi+\right. \\
& \left.+\cos ^{2} \beta \cos ^{2} \theta+\frac{1}{2} \sin 2 \beta \sin 2 \theta \sin \varphi\right), \\
B(\theta, \varphi) & =\sqrt{I_{1} I_{2}}\left(\sin \beta \sin ^{2} \theta \sin 2 \varphi+\cos \beta \sin 2 \theta \cos \varphi\right),
\end{aligned}
$$

where $I_{1,2}=A_{1,2}^{2} /\left(A_{1}^{2}+A_{2}^{2}\right)$ are the coefficients which define the part of the entire pump power contained in both incident waves.

Thus, for every molecule orientation, we have the excitation grating which is the result of polarization modulation and is described by Eq. (3). In order to calculate lasing dynamics, we use the idea of Ref. 33. Let us consider molecules of given orientation separately in maxima and minima of the excitation grating, i.e. for two values of efficiency $f_{\operatorname{exc}}^{ \pm}(\theta, \varphi)=A(\theta, \varphi) \pm|B(\theta, \varphi)|$. These two values should be substituted in the rate equations which describe temporal dynamics of the excited molecules concentration and density of generated photons. To obtain these equations, we use the rate equations given in Refs. 15, 34 for DFB laser action by intensity modulation and generalize them on the case of polarization modulation. As a result, we have the following equations for the densities $n_{ \pm}(\theta, \varphi)$ of excited molecules in maxima and minima of the excitation grating,

$$
\begin{aligned}
\frac{\mathrm{d} n_{ \pm}(\theta, \varphi)}{\mathrm{d} t} & =3 I_{p}(t) \sigma_{a} f_{\operatorname{exc}}^{ \pm}(\theta, \varphi)\left[N / 4 \pi-n_{ \pm}(\theta, \varphi)\right]- \\
& -\frac{n_{ \pm}(\theta, \varphi)}{\tau}- \\
& -3 \frac{c}{\eta} \sigma_{e} n_{ \pm}(\theta, \varphi)\left(q_{x} \cos ^{2} \varphi+q_{y} \sin ^{2} \varphi\right) \sin ^{2} \theta,
\end{aligned}
$$

where $\sigma_{a}$ and $\sigma_{e}$ are the absorption and emission crosssections; $I_{p}(t)$ is the intensity profile of the pump pulse; $q_{x}$ and $q_{y}$ are the average densities of photons polarized along $x$ and $y$ axes, respectively; $N$ is the density of dye molecules; $\tau$ is the life-time of excited state; $\eta$ is the refractive index of the dye solution; $c$ is the vacuum speed of light; the coefficient 3 is the result of usage of the cross-sections averaged over molecular orientations. The first term on the right side of Eq. (4) describes the process of molecular excitation under pump influence, while the second and third terms are responsible for spontaneous and stimulated decay of the excited state, respectively.

In order to obtain the equations for $q_{x}$ and $q_{y}$, we need the expressions for average gain coefficients for light polarized along $x$ and $y$ axes ( $\mathrm{s}-$ and $\mathrm{p}$ polarizations). These values are defined as

$k_{x, y}=\left(k_{x, y}^{+}+k_{x, y}^{-}\right) / 2$,

where the gain coefficients in the maxima and minima 
of the excitation grating can be calculated through

$k_{x}^{ \pm}=3 \sigma_{e} \int_{0}^{\pi} \int_{0}^{2 \pi} n_{ \pm}(\theta, \varphi) \cos ^{2} \varphi \sin ^{3} \theta \mathrm{d} \varphi \mathrm{d} \theta$,

$k_{y}^{ \pm}=3 \sigma_{e} \int_{0}^{\pi} \int_{0}^{2 \pi} n_{ \pm}(\theta, \varphi) \sin ^{2} \varphi \sin ^{3} \theta \mathrm{d} \varphi \mathrm{d} \theta$.

Then, the equations for average densities of s- and ppolarized photons are as follows,

$\frac{\mathrm{d} q_{x, y}}{\mathrm{~d} t}=\frac{c}{\eta} k_{x, y} q_{x, y}-\frac{q_{x, y}}{\tau_{c}^{x, y}}+\frac{\Omega}{\tau} \bar{n}$.

Here, the photon life-time in the cavity $\tau_{c}^{x, y}=$ $\eta L^{3} \alpha_{x, y}^{2} / 8 c \pi^{2}$ is proportional to squared amplitude of the gain grating [15] which, in our case, is equal to $\alpha_{x, y}=\left(k_{x, y}^{+}-k_{x, y}^{-}\right) / 2 ; L$ is the length of the DFB structure. The last term in Eq. (6) describes spontaneous emission 34 with a coefficient $\Omega=$ $b / \pi N \sigma_{a} L^{2} S$, where $b$ is the height of the excited volume, $S$ is the spectral factor which determines the fraction of spontaneous emission falling into the laser bandwidth. The average density of excited molecules is calculated by $\bar{n}=\left(<n_{+}>+<n_{-}>\right) / 2$ with $<n_{ \pm}>=\int_{0}^{\pi} \int_{0}^{2 \pi} n_{ \pm}(\theta, \varphi) \sin \theta \mathrm{d} \varphi \mathrm{d} \theta$.

Finally, lasing power can be determined by the expression [15, 34

$P_{\text {out }}=P_{x}(t)+P_{y}(t)=\frac{1}{2} \frac{h c}{\lambda_{g}} L b a\left[\frac{q_{x}(t)}{\tau_{c}^{x}(t)}+\frac{q_{y}(t)}{\tau_{c}^{y}(t)}\right]$,

where $h$ is the Planck constant, and the penetration depth $a$ of pump beam into the dye solution can be estimated from

$\frac{1}{a}=3 \sigma_{a} \frac{N}{4 \pi} \int_{0}^{\pi} \int_{0}^{2 \pi} A(\theta, \varphi) \sin \theta \mathrm{d} \varphi \mathrm{d} \theta=\sigma_{a} N$.

Equations (4) and (6) are the main relations of our model. Solving them numerically and substituting this solution into Eq. (17), we can describe dynamics of power and the polarization properties of DFB lasing by polarization modulation.

\section{Numerical results}

Let us consider some results of numerical solution of the rate equations presented in the previous section. Calculations were performed for the set of parameters which can be considered typical for the dye-laser systems: pump wavelength $\lambda_{P}=532 \mathrm{~nm}$, laser wavelength $\lambda_{L}=565 \mathrm{~nm}$, absorption and emission cross-sections (Rhodamine $6 \mathrm{G}$ ) $\sigma_{a}=3.8 \cdot 10^{-16} \mathrm{~cm}^{2}$ and $\sigma_{e}=2.15 \cdot 10^{-16} \mathrm{~cm}^{2}$, concentration of the dye solution $C=0.243 \mathrm{mM}$, refractive index of the solution at the laser wavelength $\eta=1.36$, excited state life-time $\tau=4 \mathrm{~ns}$, the length and the height of DFB structure $L=1 \mathrm{~cm}$ and $b=0.01 \mathrm{~cm}$, respectively, spectral factor $S=10^{4}$. The pulse of pump intensity has the Gaussian envelope with FWHM 0.5 ns. The ratio of intensities of pump beams with s- and p-polarizations is $2: 1$, i.e. the intensity parameters are $I_{1}=0.67$ and $I_{2}=0.33$. The angle of incidence for pump beams is $\beta=\arcsin \lambda_{P} / \lambda_{L}=70.32^{\circ}$.

We start our analysis with the laser power dynamics calculated at different pumping levels. We are interested rather in qualitative relations than in absolute values of laser characteristics. Therefore, we measure pump energy $W_{\mathrm{p}}$ with normalized parameter $\gamma=W_{\mathrm{p}} / W_{\mathrm{th}}$, where $W_{\mathrm{th}}$ is the threshold pump energy. This threshold is determined as the point of abrupt rise of the output power. In absolute units, our calculations give $W_{\mathrm{th}}=2.5 \mu \mathrm{J}$ for the parameters listed above.

According to our calculations, only s-polarized light is generated at low excesses over pump threshold. In this case, laser radiation forms a single pulse which, at threshold, appears at the trailing edge of the pump pulse and arises more and more earlier as the pump energy grows. The example of such s-polarized pulse is shown in Fig. [2(a) at $\gamma=1.28$. This singlepulse lasing corresponds to the sharp decrease of the average density $\bar{n}$ of excited molecules presented in the same figure (dotted curve). It is seen that, at first, the value $\bar{n}$ grows smoothly as a result of pump and then, when it becomes larger than certain level (which, in general, depends on pump energy), the density of excited molecules drops releasing the stored energy in the form of laser radiation. As to generation of p-polarized light under these conditions, it has low intensity and cannot be described by the characteristic bell-shaped envelope [see the lower panel in Fig. 2(a)].

When $\gamma \gtrsim 1.5$, generation of the second pulse starts. This second pulse is p-polarized that is illustrated by the profiles calculated at $\gamma=2$ [Fig. $2(b)$. In this case, the dynamics of average density of excited molecules has two well-pronounced peaks which correspond to both laser pulses. The third pulse appears for $\gamma \gtrsim 2.25$ and is s-polarized as the first one. The example of calculated power profiles and behavior of $\bar{n}$ with three maxima are shown in Fig. 2(c) for $\gamma=2.76$. Finally, when $\gamma \gtrsim 3$, the fourth pulse is generated. As can be seen in Fig. 2(d), at $\gamma=4$, this fourth pulse contains comparable quantities of both sand p-polarized radiation.

After these examples, we should consider the energetic characteristics of laser action described by our model. Figure 3 demonstrates the full output energy and the energy of s- and p-polarized laser light taken separately as the functions of pump energy. One can see that appearance of subsequent pulses is characterized by inflection points in the curves depicted 

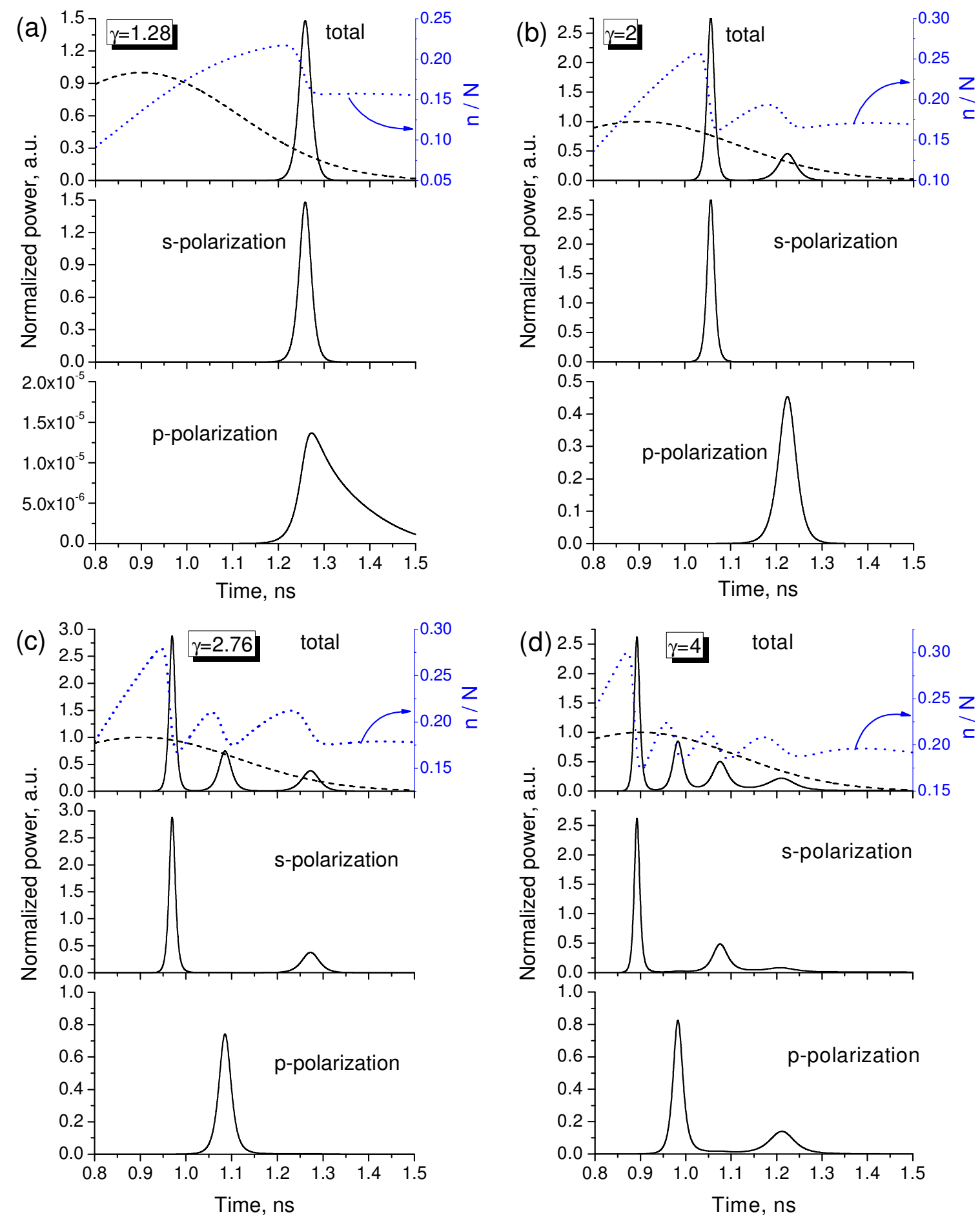

Figure 2. (Colour online) Results of calculation of the generated pulses profiles (solid lines) and dynamics of average density of excited molecules (dotted lines) at different pump levels: (a) $\gamma=1.28$, (b) 2, (c) 2.76, (d) 4. The density of excited molecules is normalized by the full density of dye molecules $N$. Dashed line shows the profile of the pump pulse.

in Fig. 3. Note that before such inflection point is reached, the plateau occurs where generated energy changes very slightly with pump energy. This property of the laser system considered seems to be perspective to obtain linearly polarized single pulses with high stability of output energy. 


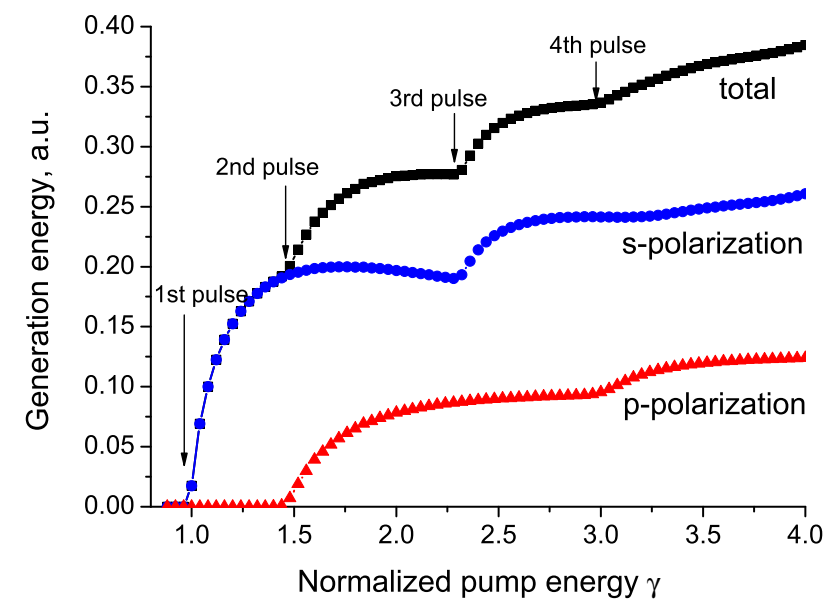

Figure 3. (Colour online) Laser output energy vs. pump energy calculated with our model. The arrows indicate pump energies where new pulse appears at the laser output.

\section{Experimental results}

The output characteristics of a DFB dye laser based on the dynamic polarization grating were investigated under subnanosecond excitation. The optical scheme of a DFB laser and registering apparatus were practically the same as in 35, 36 (Fig. 44). A $0.26 \mathrm{mM}$ ethanol solution of Rhodamine $6 \mathrm{G}$ was used as active medium. The vertically polarized second harmonic generation $\left(\lambda=532 \mathrm{~nm} ; \Delta \lambda_{0.5}<0.003 \mathrm{~nm}\right.$; beam quality factor $M^{2}<1.2$ ) from a DPSS STA01SH-500 Nd:LSB microlaser (Standa Ltd., Lithuania) delivering $\sim 0.5 \mathrm{~ns}(\mathrm{FWHM})$ pulses with an energy of $E_{P} \leq 80$ $\mu \mathrm{J}$ and a repetition rate as high as $f=500 \mathrm{~Hz}$ was employed as a pump source. The DFB laser oscillator represented a right-angle isosceles K-8 glass prism hypotenuse side of which is in optical contact with a dye in a cell with nonparallel windows. In such a scheme, the $532 \mathrm{~nm}$ pump beams are symmetrically coupled into the active medium through the side faces of the prism thereby resulting in the first-order DFB laser operation. Laser oscillations occur at a wavelength $\lambda_{L}$ given by

$\lambda_{L}=\frac{n_{s} \lambda_{P}}{2 n_{\mathrm{pr}} \sin \alpha}$,

where where $n_{s}$ is the refractive index of the dye solution at the lasing wavelength $\lambda_{L}, n_{\mathrm{pr}}$ is the refractive index of the prism material at the pump wavelength $\lambda_{P} ; \alpha$ is the incident angle of the pump beam at the prism-dye solution interface (it differs from the angle $\beta$ inside the solution). To obtain a pair of pump beams with s- and p-polarizations, respectively, the electric field vector of one of the two microlaser beams was turned by $90^{\circ}$ with the help of a half-wave plate. The intensity of s-polarized beam was approximately two times higher than that of p-polarized one. The maximum total pump beams energy falling onto the surface of the dye solution in a cell did not exceed $E_{P}=35 \mu \mathrm{J}$.

To provide optimal pumping geometry, the output beam from a microlaser with an initial diameter of about $0.02 \mathrm{~cm}$ and a full-angle divergence of $\sim 10 \mathrm{mrad}$ was collimated in the vertical plane by a cylindrical lens (focus length $18 \mathrm{~cm}$ ), while the distance between the microlaser head and the DFB laser input window was set to $\sim 99 \mathrm{~cm}$. Upon such conditions, the excited zone of the dye in a cell represented a narrow horizontal stripe with $\mathrm{a} \sim 0.9$ to $1.2 \mathrm{~cm}$ length and $\sim 0.01 \mathrm{~cm}$ height, depending on the intersection angle $2 \alpha$ of the pump beams.

During experiments, the pump input and the DFB laser output energies were measured simultaneously with calibrated FD-24K photodiodes and an ADC2OM/10-2 two-channel analog-to-digital converter (ADC). To adjust the pump power in a continuous manner, a rotatable half-wave plate in combination with a dielectric polarizer was employed. A fiber-coupled S3804 automated diffraction grating spectrograph (spectral resolution up to $\sim 0.08 \mathrm{~nm}$ ) and an IT51-30 Fabry-Perot interferometer were used for coarse and fine spectral measurements, respectively. The transient behavior of the pump and DFB laser pulses was studied using an Agat-SF3 ultrafast streak camera (time resolution up to $\sim 2$ ps). Both the interferograms and streak camera images were captured by means of a SDU-R205 charge-coupled device (CCD) USB-TV camera with subsequent computer acquisition and processing of the obtained data.

Under mentioned above pumping conditions, a DFB laser exhibited smooth wavelength tunability within $549-592 \mathrm{~nm}$ upon a spectral linewidth of $\Delta \lambda_{0.5}<0.008 \mathrm{~nm}$ and an optical-to-optical energy conversion efficiency up to $\eta_{\max } \sim 60 \%$. Dependence of the output characteristics of a DFB laser on the experimental pumping conditions was investigated in details at $\lambda_{L}=565 \mathrm{~nm}$, which falls into the dye amplification band maximum. In this case, the length of the pumped region of active medium was $L_{\mathrm{DFB}} \approx 1$ $\mathrm{cm}$.

First of all, the influence of the pump pulse intensity on the temporal course of the DFB laser output was studied in details. It was found that, generally, when pumped well above threshold, the DFB laser produces a multi-spike emission. Both an overall length and a number of spikes in the pulse train depend on the pumping level $\gamma$ and tend to decrease with decreasing the $\gamma$ value. At pumping levels not very far from threshold single ultrashort pulses of a few tens of picoseconds duration are generated.

As an example, Fig. 5 reveals streak camera traces 


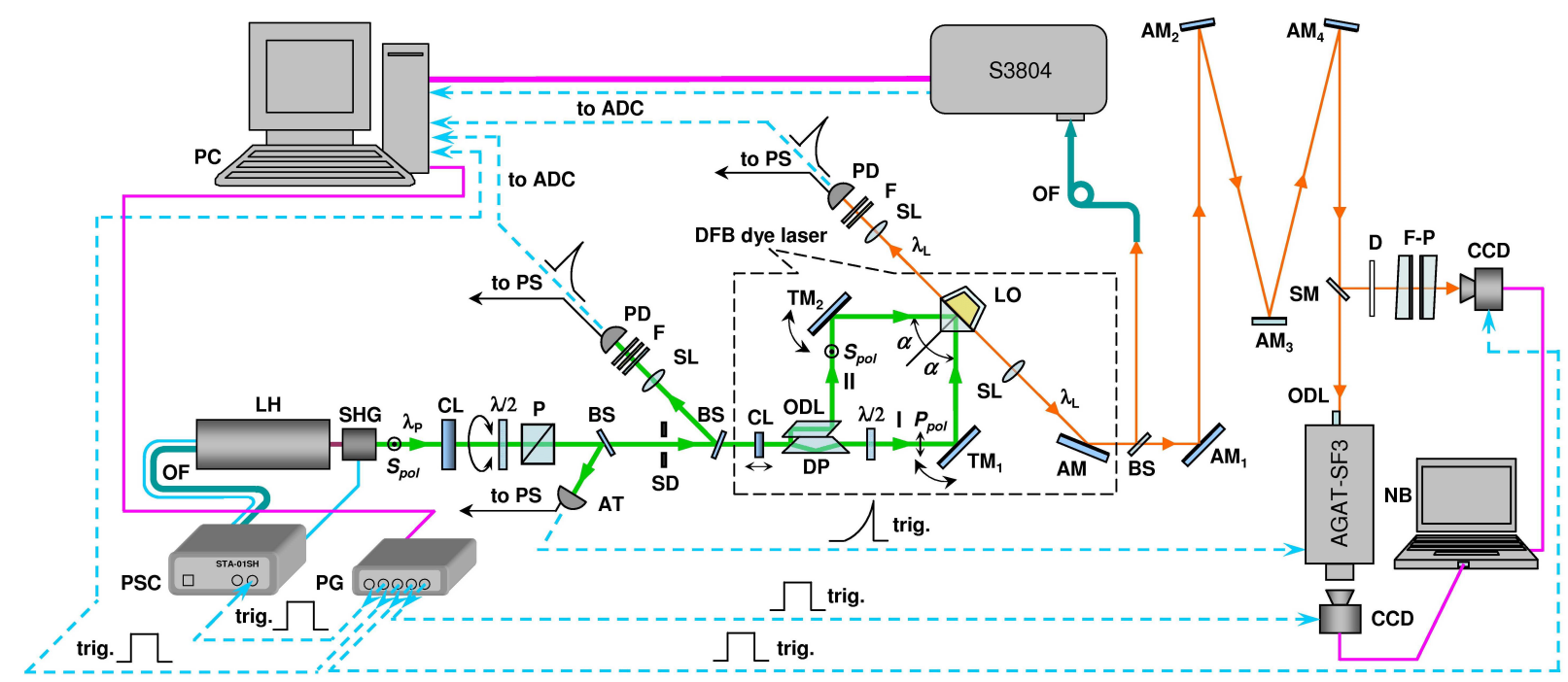

Figure 4. (Colour online) Schematic of the experimental setup. PC, personal computer; NB, notebook; S3804, automated diffraction grating spectrograph; OF, optical fiber; PSC, Nd:LSB micro laser power supply and control unit; LH, Nd:LSB micro laser head; SHG, second harmonic generator; PG, G-200P programmable TTL pulse generator; Agat-SF3, streak camera; CCD, SDU-R205 charge-coupled device USB-TV camera; PD, FD-24K silicon photodiode; AT, KT-342 hf avalanche transistor; ADC, analog-todigital converter; PS, power supply; F-P, IT51-30 Fabry-Perot interferometer; D, diffuser; CL, cylindrical lens; SL, spherical lens; F, optical filters; $\lambda / 2$, half-wave plate; $\mathrm{P}$, dielectric polarizer; BS, beam splitter; SD, slit diaphragm; DP, Dove prism beam splitter; ODL, optical delay line; TM, turning dielectric mirror; LO, DFB laser oscillator; $\alpha$, half of the pump beams I, II interference angle; $\lambda_{P}$, pumping wavelength; $\lambda_{L}$, DFB lasing wavelength; AM, aluminum mirror; SM, semi-transparent dielectric mirror.

of the DFB laser pulses registered at different pumping levels corresponding to the next pulse appearing in the laser output. Notice that, unlike Fig. 2, the zero position of the time scale is of an arbitrary character and does not reflects the real build-up dynamic of the DFB lasing. Should also be noted that, due to relatively low streak camera scan rate $\left(v_{\mathrm{sc}} \sim 1 \mathrm{~ns} / \mathrm{cm}\right)$ used during these measurements, both the individual pulses in the pulse trains and their durations are not fully resolved.

It is seen from Fig. 5(a) that at a pumping level of $\gamma \approx 3.5$ a DFB laser produces a train of four pulses with an overall width of $\sim 900$ ps. When the pump power is lowered to $\gamma \approx 3.0$ [Fig. [5(b)] and 2.2 [Fig. $5(\mathrm{c})]$, the number of emitted pulses reduces to three and two, respectively, while the overall pulse train lengths shorten to $\sim 700-800$ ps. At pumping levels $1<\gamma<1.7$ stable generation of single picosecond pulses was observed [Fig. [5(d)]. The single pulse duration was rather sensitive to the pumping level and progressively broadened with decreasing the $\gamma$ value. The shortest pulses were obtained near threshold of the second pulse $(\gamma \approx 1.7)$. In this case, their duration was measured to be about $\tau_{0.5} \approx 37$ ps (FWHM). Notice that before performing above measurements streak camera was set to operate with a scan rate of $v_{\mathrm{sc}} \sim 0.25 \mathrm{~ns} / \mathrm{cm}$ thus providing an approximately four-fold increase in the time resolution. Under such conditions, the spectral linewidth of the DFB laser emission amounted to $\Delta \lambda_{0.5} \approx 0.0075 \mathrm{~nm}$ while its energy reached $E_{L} \sim 0.13 \mu \mathrm{J}$. Based on these data, the time-bandwidth product $\nu_{0.5} \tau_{0.5}$ and peak power $P_{L}$ of a single pulse are estimated to be 0.3 and 3.5 $\mathrm{kW}$, respectively. The obtained value of $\nu_{0.5} \tau_{0.5}$ is the evidence of the transform-limited character of single picosecond pulses generated by a DFB dye laser.

Figure 6 shows the dependence of the DFB laser output energy $E_{L}$ on the pump energy $E_{P}$ measured for the range of pumping levels $1<\gamma \leq 4$ (i.e., close to that of Fig. 50. It is seen that $E_{L}$ gradually grows with increasing the pump intensity. Along with this, at least three alterations in slope may be noticed corresponding to the inflection points in Fig. 3. The first one is observed around pump energy of $\sim 1.85$ $\mu \mathrm{J}$ representing the lasing threshold, while the second and third ones correspond to the $E_{P} \approx 3.15$ and $4.1 \mu \mathrm{J}$, respectively. Based on the two latter values of pump energies, we find that the appropriate pumping levels $\gamma$ are accordingly $\sim 1.7$ and $\sim 2.2$. From streak camera measurements presented in Fig. 5(d) and (c) follows that the specified pumping level of $\gamma \approx 1.7$ and 2.2 are indicative of the second and third picosecond pulses appearing in the DFB laser output.

The above features regarding the course of the input-output characteristic of a DFB laser operating not far from threshold seem to be of undoubted practical interest. Indeed, based solely on the energy measurement, it becomes possible without using a high-cost streak camera to precisely determine the range of pump intensities within which single 


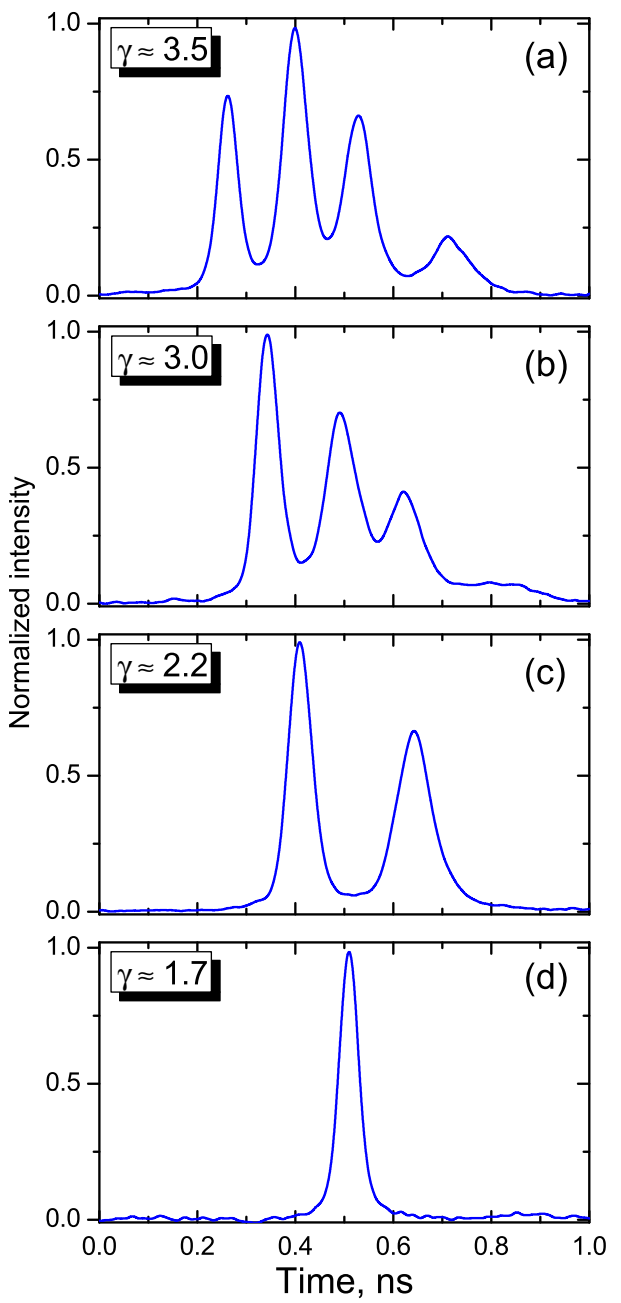

Figure 5. (Colour online) Streak camera traces of the DFB dye laser pulses taken at different pumping levels $\gamma$.

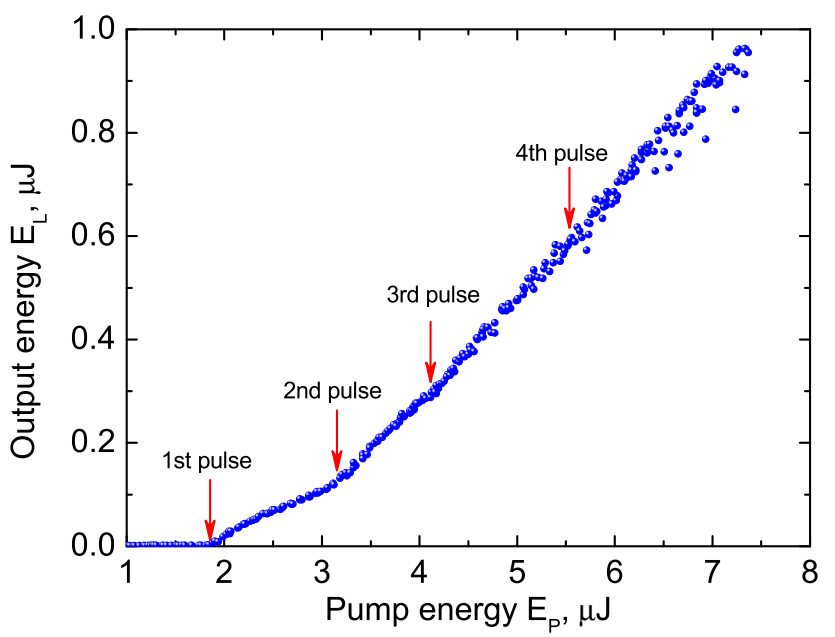

Figure 6. (Colour online) DFB dye laser output energy as a function of pump energy.

picosecond pulse generation is provided by a DFB dye laser.

\section{Conclusion}

In conclusion, we have proposed the theoretical model of DFB dye laser action by polarization modulation. This fundamentally simple model based on consideration of values averaged over excitation grating allows to describe lasing dynamics, energetic and polarization characteristics of such lasers. The mathematical basis of the model is the modified rate equations for the density of excited molecules and the concentration of laser photons. Comparison of our calculation results with experimental data shows good qualitative agreement between theory and experiment. In particular, the model allows to describe the multispike lasing and approximately predict the threshold values for successive pulse generation. Polarization characteristics of such DFB lasers will be studied in detail in a separate work.

As far as we know, we have performed the first realistic description of lasing dynamics of DFB dye laser based on polarization modulation. However, to obtain not only qualitative, but also quantitative agreement between calculations and measurements, one needs an advanced theory. In future, we plan to develop a semiclassical treatment of such lasers which is expected to give closer fit to the experimental data.

\section{Acknowledgements}

The work was supported by Belarusian State Foundation for Fundamental Research (Project F15-042).

\section{References}

[1] Kogelnik H and Shank C V 1971 Appl. Phys. Lett. 18152.

[2] Shank C V, Bjorkholm J E and Kogelnik H 1971 Appl. Phys. Lett. 18395.

[3] Chandra S, Takeuchi N and Hartman S R 1972 Appl. Phys. Lett. 21144.

[4] Zlenko A A, Prokhorov A M and Sychugov V A 1973 JETP Lett. 1891.

[5] Efendiev T Sh and Rubinov A N 1974 J. Appl. Spectr. 21 1242.

[6] Bakos J S, Fuzessy Z, Sorlei Zs and Szigeti J 1974 Phys. Lett. A 50227.

[7] Efendiev T Sh and Rubinov A N 1975 Sov. J. Quantum Electron. 5478.

[8] Rubinov A N, Efendiev T Sh, Adamushko A V and Bor J 1977 Opt. Commun. 20159.

[9] Rubinov A N and Efendiev T Sh 1977 J. Appl. Spectr. 27 1275.

[10] Bor Zs, Racz B, Kozma L, Rubinov A N and Efendiev T Sh 1978 Opt. Commun. 24265.

[11] Rubinov A N and Efendiev T Sh 1985 Opt. Acta 321291.

[12] Zaporozhchenko V A, Rubinov A N and Efendiev T Sh 1977 Pisma ZhTF 3114.

[13] Bushuk B A, Zaporozhchenko V A, Rubinov A N and Efendiev T Sh 1979 Pisma ZhTF 5880.

[14] Bor Zs 1979 Opt. Commun. 29103.

[15] Bor Zs 1980 IEEE J. Quant. Electron. 16517. 
[16] Masilamani V, Pozzi L and Docchio F 1985 Appl. Phys. B 3735.

[17] Efendiev T Sh, Katarkevich V M and Rubinov A N 1985 Opt. Commun. 55347.

[18] Ermilov E A and Gulis I M 2001 Quantum Electron. 31 857.

[19] Diao Z, Deng S, Huang W, Xuan L, Hu L., Liu Y and Ma J 2012 J. Mater. Chem. 2223331.

[20] Toffanin S, Kim S, Cavallini S, Natali M, Benfenati V, Amsden J J, Kaplan D L, Zamboni R, Muccini M and Omenetto F G 2012 Appl. Phys. Lett. 101091110.

[21] Chida T and Kawabe Y 2012 Proc. SPIE 8464 84640E.

[22] Vannahme C, Maier-Flaig F, Lemmer U and Kristensen A 2013 Lab Chip 132675.

[23] Goldenberg L M, Lisinetskii V, Gritsai Yu, Stumpe J and Schrader S 2013 Laser Phys. Lett. 10085804.

[24] Da Silva R R, Dominguez C T, dos Santos M V, BarbosaSilva R, Cavicchioli M, Christovan L M, de Melo L S A, Gomes A S L, de Araujo C B and Ribeiro S J L $2013 \mathrm{~J}$. Mater. Chem. C 17181.

[25] Smirnova T N, Sakhno O V, Fitio V M, Gritsai Yu and Stumpe J 2014 Laser Phys. Lett. 11125804.

[26] Sakhno O V, Gritsai Yu and Stumpe J 2014 Laser Phys. Lett. 11115812.

[27] Lo D, Ye C and Wang J 2003 Appl. Phys. B 76649.

[28] Ye C, Wang J, Shi L and Lo D 2004 Appl. Phys. B 78189.

[29] Wang J, Dong H, Fan J, Li R, Zhang L and Wong K Y 2011 Appl. Opt. $\mathbf{5 0} 6248$.

[30] Chen F 2013 Opt. Commun. 294260.

[31] Katarkevich V M, Rubinov A N and Efendiev T Sh 2013 Proc. IV Congress of Physicists of Belarus (Minsk) p. 84.

[32] Katarkevich V M, Rubinov A N and Efendiev T Sh 2013 Proc. IX Int. Conf. Quant. Electron. (Minsk) p. 90.

[33] Rubinov A N, Rubinov Ya A, Katarkevich V M and Efendiev T Sh 2000 J. Appl. Spectr. 67990.

[34] Bor Zs, Müller A, Racz B and Schäfer F P 1982 Appl. Phys. B 279.

[35] Efendiev T Sh, Katarkevich V M, Qajar Ch O and Karamaliyev R A 2011 Azerb. J. Phys. 177.

[36] Katarkevich V M, Rubinov A N, Efendiev T Sh, Anufrik S S and Koldunov M F 2015 Appl. Opt. 547962. 